EPJ manuscript No.

(will be inserted by the editor)

\title{
A fully relativistic description of Hypernuclear production in proton- and pion-Nucleus Collisions
}

\author{
R. Shyam ${ }^{1}$ a \\ Saha Institute of Nuclear Physics, 1/AF Bidhan Nagar, Kolkata, India \\ Received: date / Revised version: date
}

\begin{abstract}
Exclusive $A\left(p, K^{+}\right)_{\Lambda} B$ and $A\left(\pi, K^{+}\right)_{\Lambda} B^{\prime}$ reactions leading to two body final states, have been investigated in a fully covariant model based on an effective Lagrangian picture. The explicit kaon production vertex is described via creation, propagation and decay into relevant channel of $N^{*}(1650), N^{*}(1710)$ and $N^{*}(1720)$ intermediate baryonic resonance states, in the initial collision of the projectile with one of the target nucleons. The bound state wave functions are obtained by solving the Dirac equation with appropriate scalar and vector potentials. The calculated cross sections show strong sensitivity to the final hypernuclear state excited in the reaction. Cross sections of $1-2 \mathrm{nb} / \mathrm{sr}$ are obtained at peak positions of favored transitions in case of the $A\left(p, K^{+}\right){ }_{\Lambda} B$ reaction on heavier targets.
\end{abstract}

PACS. PACS-key 25.40.Ve - PACS-key 13.75.-n,

\section{Introduction}

$\Lambda$ hypernuclei have been studied extensively by stopped as well as in-flight $\left(K^{-}, \pi^{-}\right)$[1,2] and $\left(\pi^{+}, K^{+}\right)$reactions [3. The kinematical properties of the $\left(K^{-}, \pi^{-}\right)$reaction allow only a small momentum transfer to the nucleus (at forward angles), thus there is a large probability ' of populating $\Lambda$-substitutional states in the residual hypernucleus. On the other hand, in the $\left(\pi^{+}, K^{+}\right)$reaction the momentum transfer is larger than the nuclear Fermi momentum, therefore, hypernuclear states with configurations of an outer neutron hole and a $\Lambda$ hyperon in a series of orbits covering all bound states can be excited in this case. The data on the hypernuclear spectroscopy have been used extensively to extract information about the hyperon-nucleon interaction within a variety of theoretical approaches (see, e.g., 4,5).

Alternatively, $\Lambda$-hypernuclei can also be produced with high intensity proton beams via the $\left(p, K^{+}\right)_{\Lambda} B$ reaction where the hypernucleus ${ }_{\Lambda} B$ has the same neutrons and proton numbers as the target nucleus $A$. First set of data for this reaction on deuterium and helium targets, have already been reported in Ref. [6]. The states of the hypernucleus excited in the $\left(p, K^{+}\right)$reaction may have a different type of configuration as compared to those excited in the $\left(\pi^{+}, K^{+}\right)$reaction. Thus a comparison of informations extracted from the study of two reactions is likely to lead to a better understanding of the hypernuclear structure.

Theoretical studies of the $A\left(p, K^{+}\right)_{\Lambda} B$ reaction are based on two main approaches; the one-nucleon model

\footnotetext{
a Present address:Institut für Theoretische Physik, Universität Giessen, D-35392 Giessen
}

(ONM) [Fig. 1(a)] and the two-nucleon model (TNM) [Fig. 1(b) and 1(c)]. In the ONM the incident proton first scatters from the target nucleus and emits a (off-shell) kaon and a $\Lambda$ hyperon which gets captured into one of the (target) nuclear orbits. Thus there is only one single active nucleon (impulse approximation) which carries the entire momentum transfer to the target nucleus (about 1.0 $\mathrm{GeV} / \mathrm{c}$ at the outgoing $K^{+}$angle of $0^{\circ}$ ). This makes this model extremely sensitive to details of the bound state wave function at very large momenta where its magnitude is very small leading to quite low cross sections. In the ONM calculations of $\left(p, K^{+}\right)$and also of $(p, \pi)$ reactions the distortion effects in the incident and the outgoing channels have been found to be quite important [7,8].

In the two-nucleon mechanism (TNM), the kaon production proceeds via a collision of the projectile nucleon with one of its target counterparts. This excites intermediate baryonic resonant states which decay into a kaon and a $\Lambda$ hyperon. The nucleon and the hyperon are captured into the respective nuclear orbits while the kaon rescatters into its mass shell. In this picture there are altogether three active bound state baryon wave functions taking part in the reaction process allowing the large momentum transfer to be shared among three baryons. Consequently, the sensitivity of the model is shifted from high momentum to the lower momentum parts of the bound state wave functions where they are relatively better known.

A fully covariant TNM for the $A\left(p, K^{+}\right)_{\Lambda} B$ reaction has been developed in Refs 9,10 by retaining the field theoretical structure of the interaction vertices and by treating the baryons as Dirac particles. The initial interaction between the incoming proton and a bound nucleon 


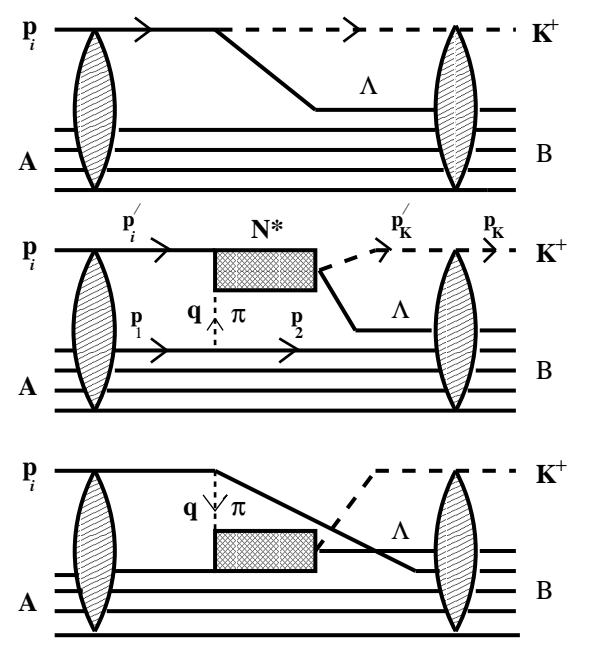

(a)

Fig. 1. Graphical representation of one- and two-nucleon models of $A\left(p, K^{+}\right)_{\Lambda} B$ reaction. The elliptic shaded area represent the optical model interactions in the incoming and outgoing channels.

of the target is described by $\pi, \rho$ and $\omega$ exchange mechanisms. This leads to the excitations of $N^{*}(1650)\left[\frac{1}{2}^{-}\right]$, $N^{*}(1710)\left[\frac{1}{2}^{+}\right]$, and $N^{*}(1720)\left[\frac{3}{2}^{+}\right]$baryonic resonance intermediate states which decay into kaon and the $\Lambda$ hyperon. We present a brief sketch of this formalism in the next section.

\section{Covariant Two-nucleon Model}

We provide here only a brief outline of our TNM; details of this theory are given in Refs. [9, 10. The structure of the TNM for the $\left(p, K^{+}\right)$reaction is similar to that of the effective Lagrangian approach for the elementary $p p \rightarrow p \Lambda K^{+}$process as discussed in Ref.[11. We use the same effective Lagrangians and vertex parameters to model the initial interaction between the incoming proton and a bound nucleon of the target by means of $\pi, \rho$ and $\omega$ exchange mechanisms. The structure for the resonancenucleon-meson vertices were also taken to be the same. Terms corresponding to the interference between various amplitudes are retained. After having established the effective Lagrangians and the coupling constants for various vertices the amplitudes for the graphs $1 \mathrm{~b}$ and $1 \mathrm{c}$ can be written by following the well known Feynman rules. These amplitudes can be evaluated by following the techniques described in Ref. [10. It should be noted that the Fig. 1c can be used in a straight forward way to calculate the amplitudes of the $A\left(\pi, K^{+}\right)_{\Lambda} B^{\prime}$ reaction.

The differential cross section for the $\left(p, K^{+}\right)$reaction is given by

$$
\frac{d \sigma}{d \Omega}=\frac{1}{(4 \pi)^{2}} \frac{m_{p} m_{A} m_{B}}{\left(E_{p_{i}}+E_{A}\right)^{2}} \frac{p_{K}}{p_{i}} \sum_{m_{i} m_{f}}\left|T_{m_{i} m_{f}}\right|^{2},
$$

where $E_{p_{i}}$ and $E_{A}$ are the total energies of incident proton and the target nucleus, respectively while $m_{p}, m_{A}$ and $m_{B}$

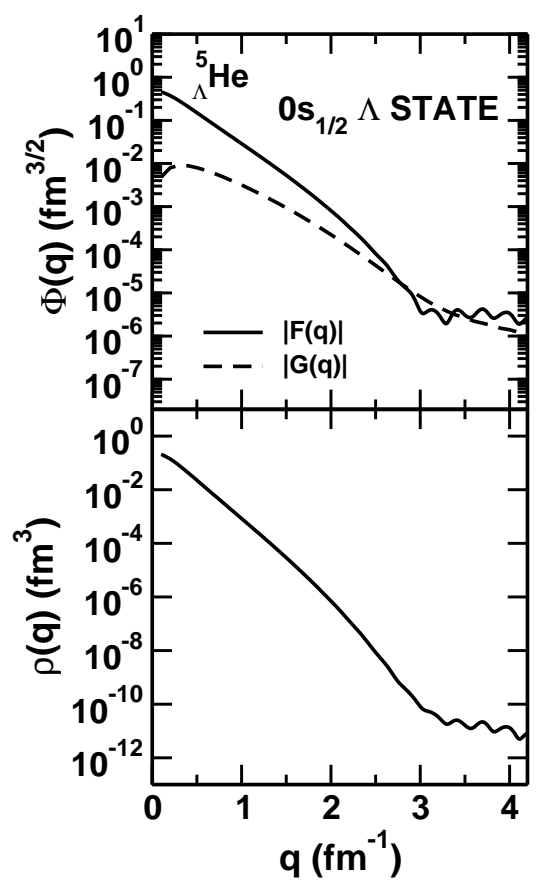

Fig. 2. (Upper panel) Momentum space spinors $[\Phi(q)]$ for $0 s_{1 / 2}$ $\Lambda$ orbit in ${ }_{\Lambda}^{5} \mathrm{He}$ hypernucleus. $|F(q)|$ and $|G(q)|$ are the upper and lower components of the spinor, respectively. (Lower panel) Momentum distribution $\left[\rho(q)=|F(q)|^{2}+|G(q)|^{2}\right]$ for the same hyperon state calculated with these wave functions.

are the masses of the proton, and the target and residual nuclei, respectively. The summation is carried out over initial $\left(m_{i}\right)$ and final $\left(m_{f}\right)$ spin states. $T$ is the final $T$ matrix obtained by summing the transition

\section{Results and Discussions}

The spinors for the final bound hypernuclear state (corresponding to momentum $p_{\Lambda}$ ) and for two intermediate nucleonic states (corresponding to momenta $p_{1}$ and $p_{2}$ ) are required to perform numerical calculations of various amplitudes. We assume these states to be of pure-single particle or single-hole configurations with the core remaining inert. The spinors in the momentum space are obtained by Fourier transformation of the corresponding coordinate space spinors which are the solutions of the Dirac equation with potential fields consisting of an attractive scalar part $\left(V_{s}\right)$ and a repulsive vector part $\left(V_{v}\right)$ having a Woods-Saxon form. The potential parameters are given in Ref. [10].

In Figs. 2 and 3, we show the lower and upper components of the Dirac spinors in momentum space for the $0 s_{1 / 2}$ hyperon in ${ }_{4}^{5} \mathrm{He}$ and the $0 p_{3 / 2}$ and $0 s_{1 / 2}$ hyperons in ${ }_{1}^{13} \mathrm{C}$, respectively. In each case, we note that only for momenta $<1.5 \mathrm{fm}^{-1}$, is the lower component of the spinor substantially smaller than the upper component. In the region of momentum transfer pertinent to exclusive kaon production the lower components of the spinors are not 


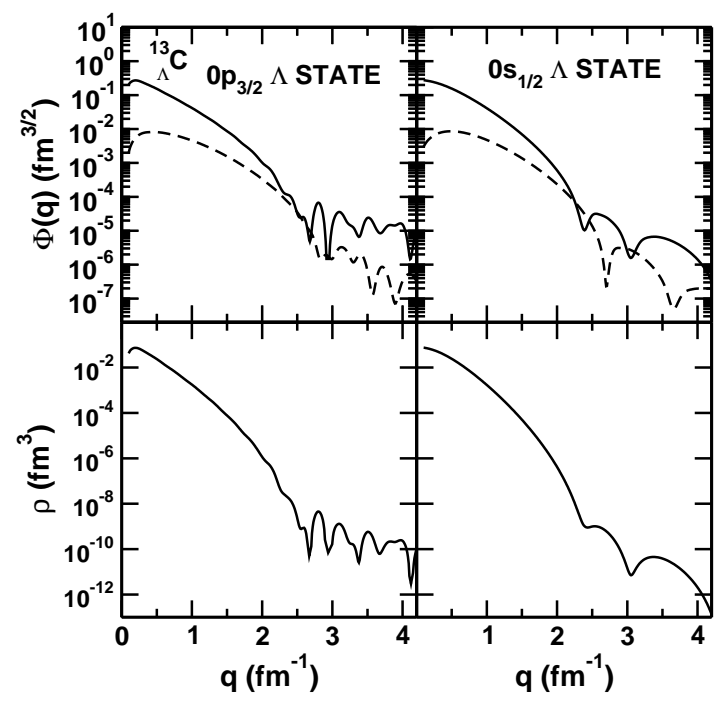

Fig. 3. same as in Fig. 2 for $0 p_{3 / 2} \Lambda$ and $0 s_{1 / 2} \Lambda$ orbits in hypernucleus ${ }_{\Lambda}^{13} C$.

negligible as compared to the upper component which demonstrates that a fully relativistic approach is essential for an accurate description of this reaction. The spinors calculated in this way provide a good description of the experimental nucleon momentum distributions for various nucleon orbits as is shown in Ref. [12. In the lower panel of each of Figs. 2 and 3 we show momentum distribution $\rho(q)\left[=\left.F(q)\right|^{2}+|G(q)|^{2}\right]$ of the corresponding $\Lambda$ hyperon. In each case the momentum density of the hyperon shell, in the momentum region around $0.35 \mathrm{GeV} / \mathrm{c}$, is at least 2-3 orders of magnitude larger than that around 1.0 $\mathrm{GeV} / \mathrm{c}$. In the TNM, therefore cross section will be larger than the those in the ONM as in the former case the sensitivity of the model gets shifted to lower momenta due to involvement of three baryon in the momentum sharing process.

A crucial quantity needed in the calculations of the kaon production amplitude is the pion self-energy, $\Pi(q)$, which takes into account the medium effects on the intermediate pion propagation. Since the energy and momentum carried by such a pion can be quite large (particularly at higher proton incident energies), a calculation of $\Pi(q)$ within a relativistic approach is mandatory. In our study contributions from the particle-hole $(p h)$ and delta-hole $(\Delta h)$ excitations are taken into account. The self-energy has been renormalized by including the short-range repulsion effects by introducing the constant Landau-Migdal parameter $g^{\prime}$ which is taken to be the same for $p h-p h$ and $\Delta h-p h$ and $\Delta h-\Delta h$ correlations which is a common choice.

In Fig. 4, we show the dependence of our calculated cross sections on pion self-energy. It is interesting to note that this has a rather large effect. We also note a surprisingly large effect on the short range correlation (expressed schematically by the Landau- Migdal parameter $g^{\prime}$ ). Similar results have also been reported in case of the

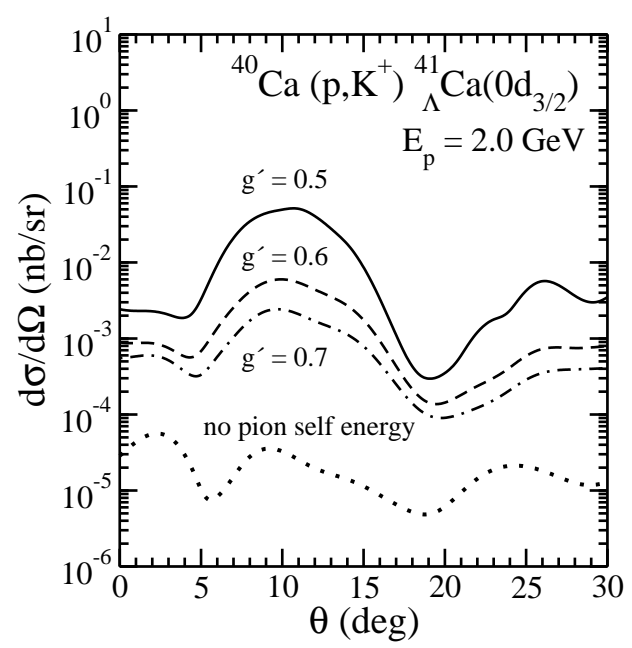

Fig. 4. Differential cross section for the ${ }^{40} \mathrm{Ca}\left(p, K^{+}\right){ }_{\Lambda}^{41} \mathrm{Ca}\left(0 d_{3 / 2}\right)$ reaction for the incident proton energy of $2.0 \mathrm{GeV}$. The dotted line shows the results obtained without including the pion self-energy in the denominator of the pion propagator while full, dashed and dashed-dotted lines represent the same calculated with pion self-energy renormalized with Landau-Migdal parameter of 0.5, 0.6 and 0.7 , respectively.

$(p, \pi)$ reactions. However, more definite statements about the usefulness of $\left(p, K^{+}\right)$reactions in probing the medium effects on the pion propagation in nuclei must await the inclusions of distortions in the incident and outgoing channels.

In Figs. 5 and 6, we show the kaon angular distributions for various final hypernuclear states excited in ${ }^{12} \mathrm{C}\left(p, K^{+}\right){ }_{\Lambda}^{13} \mathrm{C}$ and ${ }^{4} \mathrm{He}\left(p, K^{+}\right){ }_{\Lambda}^{5} \mathrm{He}$ reactions, respectively. The incident proton energies in the two cases are taken to be $1.8 \mathrm{GeV}$ and $2.0 \mathrm{GeV}$, respectively where the angle integrated cross sections for the two reactions are maximum. The calculations are the coherent sum of all the amplitudes corresponding to the various meson exchange processes and intermediate resonant states. Clearly, the cross sections are quite selective about the excited hypernuclear state, being maximum for the state of largest orbital angular momentum. This is due to the large momentum transfer involved in this reaction.

It should be noted that in in Fig. 2 the angular distribution has a maximum at angles larger than $0^{\circ}$. In contrast to this, the maximum in Fig 3, occurs at zero degree and it decreases gradually as the angle increases. This is due to the fact that in the bound state spinors of ${ }_{1}^{13} \mathrm{C}$, there are several maxima in the upper and lower components of the momentum space bound spinors in the region of large momentum transfers. Therefore, in the kaon angular distribution the first maximum may shift to larger angles reflecting the fact that the bound state wave functions show diffractive structure at higher momentum transfers. On the other hand, for momentum transfers relevant to ${ }^{4} \mathrm{He}\left(p, K^{+}\right){ }_{4}^{5} \mathrm{He}$ reaction the Dirac spinors are smoothly varying and are devoid of structures as can be seen in Fig. 2. In any case, from the purely kinematical arguments 


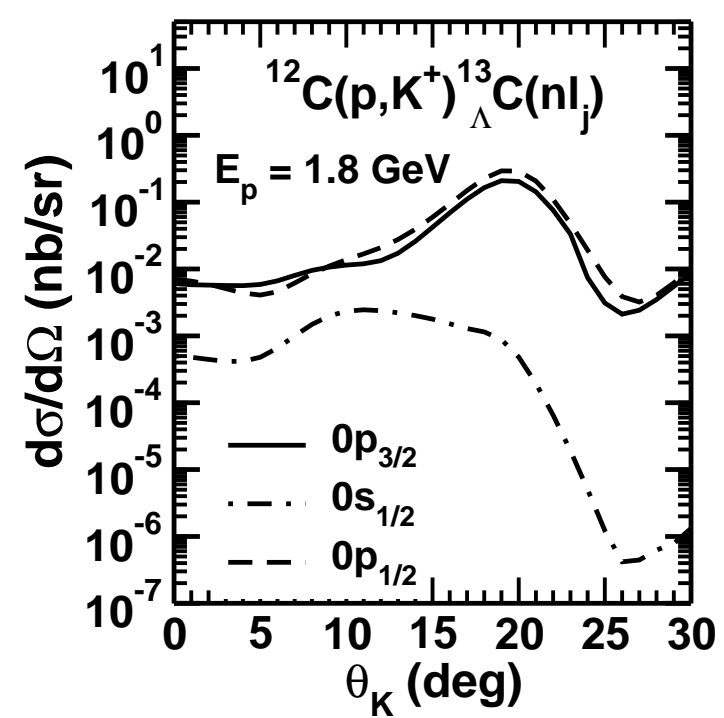

Fig. 5. Differential cross section for the ${ }^{12} C\left(p, K^{+}\right){ }_{\Lambda}^{13} C$ action for the incident proton energy of $1.8 \mathrm{GeV}$ for various bound states of final hypernucleus as indicated in the figure.

it is clear that the maximum in the cross section for a $\ell=0$ transition is expected to occur at smaller angles as compared that for a $\ell \neq 0$ one.

\section{Summary and Conclusions}

In summary, we have made a study of the $A\left(p, K^{+}\right)_{\Lambda} B$ reaction on ${ }^{4} \mathrm{He},{ }^{12} \mathrm{C}$, and ${ }^{40} \mathrm{Ca}$ targets and of the $A\left(p, K^{+}\right)_{\Lambda} B$ reaction on the ${ }^{12} \mathrm{C}$ target within a fully covariant general two-nucleon mechanism where in the initial collision of the projectile with one of the target nucleons, $N^{*}(1710)$, $N^{*}(1650)$, and $N^{*}(1720)$ baryonic resonances are excited which subsequently propagate and decay into the relevant channel. Wave functions of baryonic bound states are obtained by solving the Dirac equation with appropriate scalar and vector potentials.

In the case of the nucleon projectile, the cross sections are dominated by the graphs of the type shown in Fig.1b (target emission graph). The one-pion-exchange processes make up most of the differential cross section at all angles. The calculated cross sections are maximum for the hypernuclear state with the largest orbital angular momentum. For heavier targets, the angular distributions for the favored transitions peak at angles larger than the $0^{\circ}$ which in contrast to the results of most of the previous nonrelativistic calculations for this reaction. This reflects directly the nature of the Dirac spinors for the bound states which involve several maxima in the region of large momentum transfer. In case of the light target ${ }^{4} \mathrm{He}$, however, the differential cross section still peaks near the zero degree as in this case the momentum transfers are in the region where the bound state spinors are smoothly decreasing with momentum. The energy dependence of the calculated total production cross section follows closely that of the $p p \rightarrow p \Lambda K^{+}$reaction. We find that the nuclear

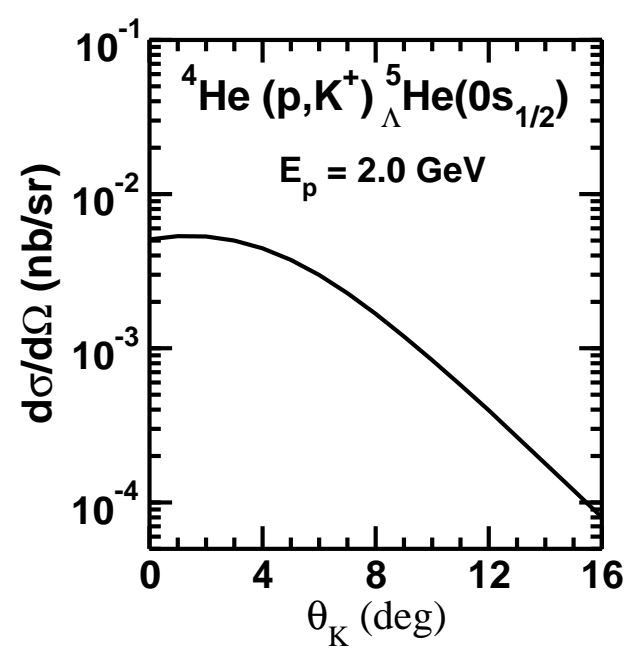

Fig. 6. Differential cross section for the ${ }^{4} \mathrm{He}\left(p, K^{+}\right){ }_{\Lambda}^{5} \mathrm{He}$ reaction for the incident proton energy of $2.0 \mathrm{GeV}$ for the bound state of final hypernucleus as indicated in the figure.

medium corrections to the intermediate pion propagator introduce large effects on the kaon differential cross sections. There is also the sensitivity of the cross sections to the short-range correlation parameter $g^{\prime}$ in the pion self-energy. Thus, $\left(p, K^{+}\right)$reactions may provide an interesting tool to investigate the medium corrections on the pion propagation in nuclei.

In case of the pion induced reaction, the hypernuclear states with stretched spin configurations are preferentially excited.

The author wishes to thank Horst Lenske and Ulrich Mosel for several useful discussions and collaboration on this subject.

\section{References}

1. R. E. Chrien and C. B. Dover, Annu. Rev. Nucl. Part. Sci. 39 (1989) 113.

2. H. Bandō, T. Motoba and J. Žofka, Int. J. Mod. Phys. 5 (1990) 4021.

3. O. Hashimoto, H. Tamura, Prog.Part.Nucl.Phys.57 (2006) 564.

4. E. Hiyama, M. Kamimura, T. Motoba, T. Yamada, and Y. Yamamoto, Phys. Rev. Lett. 85 (2000) 270

5. C. M. Keil and H. Lenske, Phys. Rev. C 66 (2002) 054307.

6. J. Kingler et al., Nucl. Phys. A634 (1998) 325.

7. B. V. Krippa, Z. Phys. A 351, (1995) 411.

8. E. D. Cooper and H. S. Sherif, Phys. Rev. Lett. 47, 818 (1982); Phys. Rev. C 25, 3024 (1982).

9. R. Shyam, H. Lenske, and U. Mosel, Phys. Rev. C 69 (2004) 065205.

10. R. Shyam, H. Lenske, and U. Mosel, Nuc. Phys. 764 (2006) 313.

11. R. Shyam, Phys. Rev. C 60 (1999) 055213.

12. R. Shyam, W. Cassing and U. Mosel, Nucl. Phys. A586 (1995) 557. 